110 Gu starson: Darst. v. Chlor-, Brom- u. Jodaluminium.

Anders als Zinkäthyl und Zinkpropyl reagirt Zinkmethyl. Die Reaction wird von Gasentwicklung, starker Erwärmung und Bildung eines voluminösen Niederschlages begleitet. Die dabei in kleiner Menge gebildeten basischen Substanzen wurden bisher von mir nicht untersucht.

Also reagiren Zinkalkyle mit (NO)- und Alkoxyl-Gruppen der Salpetrigsäureester analog, wie mit Carbonyl- und AlkoxylGruppen des Oxalsäure- ${ }^{1}$ ) und Ameisensäureester ${ }^{2}$ ), der Aldehyde ${ }^{3}$; und Carbonsäurechloranhydride. $\left.{ }^{4}\right)$

Bei der Bildung der Dinitroäthylsäure Frankland's aus Stickoxyd und Zinkäthyl tritt von zwei Molekülen Stickoxyd nur eins in die Reaction ein; es verhalten sich dabei (NO)-Gruppen analog den Carbonylgruppen von Oxalsäureester, aus dessen zwei Carbonylen nur eins mit Zinkäthyl reagirt. Die Entstehung der Dinitroäthylsäure lässt sich durch folgendes Schema veranschaulichen:

und

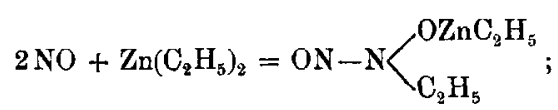

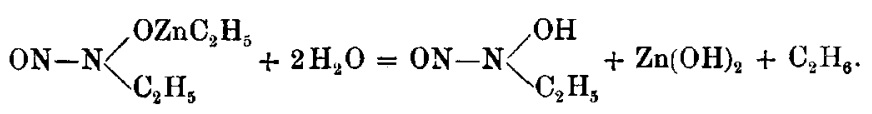

(Schluss folgt.)

\title{
Ueber die Darstellung von Chlor-, Brom- und Jodalumininm;
} von

\section{G. Gustavson.}

Die Veröffentlichung dieser Notiz ist veranlasst durch eine jüngst von $M$. Gomberg beschriebene Darstellungsmethode des Aluminiumchlorids. ${ }^{5}$ ) Ich bin ganz mit Herrn Gromberg

1) Frankland u. Duppa, Ann. Chem. 135, 25.

2) G. Wagner und Al. Saytzeff, Ann. Chem. 175, 351; Kannonnikoff u. Al. Saytzeff, Das. 175, 374.

3) G. Wagner, Journ. de la Soc. physico-chimique russe 16, 283.

$\left.{ }^{4}\right)$ Butlerow, Bull, soc. chim. (2) V, 17; (2) II, 106.

b) M. Gomberg, Ber. 33, 3146. 
einverstanden, dass man aus Aluminium und Chlor zum Aluminiumchlorid weit bequemer gelangt, als aus Aluminium und Chlorwasserstoff. ${ }^{1}$ ) Ich habe schon vor einer Reibe von Jahren die auf directe Vereinigung von Halogenen mit Aluminium basirte Darstellungsmethoden von Chlor-, Brom- und Jodaluminium veröffentlicht. Da aber meine Abhandlung in einer nicht speciell chemischen und wenig verbreiteten Zeitschrift erschien ${ }^{2}$ ), und desshalb bis jetzt den Fachgenossen wenig bekannt wurde, so erlaube ich mir, wegen einiger Einzelheiten die betreffende Stelle aus dieser Abhandlung zu reproduciren.

,Zur Bereitung von Chloraluminium leitet man trocknes Chlor durch ein Rohr von schwer schmelzbarem Glase $(4-5 \mathrm{Dcm}$. lang und $1 \frac{1}{2} \mathrm{Cm}$. weit), welches der ganzen Länge nach mit grossen Stücken Aluminiumblech angefüllt ist. Ein schwaches Erwärmen genügt, um die Reaction einzuleiten. Aluminiumstücke, die dem chlorzuführenden Rohre am nächsten liegen, erglühen in Folge der Reaction und ein weiteres Erwärmen von aussen her wird an jener Stelle dann überflüssig. Ein bequemes Verfahren zum Auffangen des Chloraluminiums besteht in Folgendem. Auf das freie Ende des Rohres, welches mit Aluminium beschickt ist, setzt man ein etwa $2 \mathrm{Dcm}$. langes Stück eines anderen Rohres auf, dessen Lichtung so weit sein muss, dass das Rohr mit Aluminium in dasselbe bequem eingeschoben werden kann. In diesem kurzen Rohre, welches als Vorlage dient, verdichten sich alsdann die Dämpfe von Chloraluminium. Ist die Menge von Chloraluminium darin so beträchtlich, dass es den Durchgang der Gase versperrt, so tauscht man das Robr gegen ein anderes von eben solchen Dimensionen aus. Wenngleich das Chloraluminium sich ausserordentlich fest an die Wände ansetzt, so lässt es sich doch leicht mit Hülfe eines starken Glasstabes aus den Röhren entfernen. Man kann die Operation leicht zu einer continuirlichen machen und grosse Mengen von Chloraluminium darstellen, ohne den Apparat auseinanderzunehmen; man braucht nur von Zeit zu Zeit neue Quantitäten von Aluminium nachzufüllen. Das Chloraluminium muss durchaus in zugeschmolzenen Gefässen aufbewahrt werden.

Auf ähnliche Weise stellte ich Bromaluminium dar. Einen gleichmässigen Strom von Brom erhält man am besten, wenn man dasselbe vermittelst eines Hahntrichters in einen im Paraffinbade $\left(100^{\circ}-120^{\circ}\right)$ befindlichen Kolben, der durch ein angeschweisstes Seitenrohr und den daran angebrachten Kaut-

1) Stockhausen u. Gattermann, Ber. 25, 3521.

3) G. Gustavs on, Die organischen Verbindungen in ihrer Beziehung zu den Haloïdsalzen des Aluminiums. Bulletin de la société Imp. des naturalistes de Moscou, 1886, No. 2. 
schukpfropfen mit dem Glasrohr verbunden, tropfenweise einfliessen lässt. Das letztere muss sich in einer gegen die Vorlage geneigten Stellung befinden; als Vorlage kann man eine Retorte, deren Hals zur Hälfte abgesprengt ist, und in die dann das freie Ende des Rohres eingeschoben wird, benutzen. Anfänglich wird das Aluminium erhitzt und dann Brom durchgeleitet; ist aber die Reaction einmal in Gang gerathen, so kann man das Erhitzen ganz einstellen; doch muss man darauf achten, dass das entstandene Bromaluminium nicht im Rohre erstarrt, sondern in die Vorlage abfliesst. Den Zufluss von Brom kann man leicht so reguliren, dass es fast vollständig in Reaction tritt. Die Vorlage abzukühlen ist überflüssig. Das erhaltene Bromaluminium muss einer Destillation unterworfen werden, am zweckmässigsten aus derselben Retorte, die bereits als Vorlage gedient hat. Die ersten Portionen des Destillates enthalten Brom und können zu Reactionen, bei denen die Gegenwart von Brom und Bromaluminium nothwendig, Verwendung finden. Die nächstfolgenden Fractionen sicd fast farblos. Bromaluminium muss ebenfalls in zugeschmolzenen Gefässen aufbewahrt werden.

Zur Bereitung von Jodaluminium aus Aluminiumdraht verfuhr ich genau so, wie ich vor zehn Jahren berichtete. ${ }^{1}$ )

Noch einfacher gestaltet sich die Bereitung von Jodaluminium, wenn man seine Lösung in Schwefelkohlenstoff darzustellen hat. Man bringt alsdann in eine Stöpselflasche Jod und Stücke von Aluminiumblech in einer zur Bildung von $\mathrm{AlJ}_{3}$ erforderlichen Menge, giesst in das Gefäss die dreifache Gewichtsmenge Schwefelkohlenstoff $\left(\mathrm{AlJ}_{3}\right.$ löst sich ungefähr in drei Theilen Schwefelkohlenstoff), verschliesst dasselbe mit dem Stöpsel, schüttelt es und lässt es in Wasser bei Zimmertemperatur stehen. Am anderen Tage schüttelt man das Gefäss von neuem, wobei eine schwache Erwärmung des Gemenges bemerkbar wird. Die Aluminiumstücke erscheinen stark zerfressen. Wieder lässt man es 24 Stunden lang stehen; nach Verlauf derselben muss die Reaction als beendet angesehen werden: es hat sich eine Lösung von Jodaluminium in Schwefelkohlenstoff gebildet. Die erhaltene Lösung ist immer durch etwas freies Jod gefärbt, selbst bei Ueberschuss von Aluminium. Auf ähnliche Weise, wenn auch langsamer, geht die Bildung der Lösung von Jodaluminium in Benzol, wie auch in Jodäthyl, vor sich."

1) Ann. Chem. 172, 173. 